\title{
INCIDENCE DES CARACTÉRISTIQUES DES ÉLÈVES SUR LEUR DEGRÉ DE MOTIVATION À PARTICIPER À UNE EXPO-SCIENCES
}

\author{
Louis Trudel ${ }^{*}$ \\ Giuliano Reis** \\ Liliane Dionne ${ }^{* * *}$
}

RÉSUMÉ: Les expo-sciences sont de plus en plus populaires chez les jeunes. Leur motivation à participer peut être influencée par différents facteurs que notre étude cherche à préciser. A cette fin, nous avons construit un questionnaire destiné à mesurer le degré de motivation des participants à l'expo-sciences pan canadienne qui regroupe chaque année les meilleurs au pays. Nos résultats indiquent que ces élèves sont très motivés mais que contrairement à certains résultats de recherches antérieures, leur degré de motivation est influencé par leur âge et leur niveau scolaire. Les autres facteurs étudiés n'ont pas eu d'influence significative sur leur degré de motivation. Nous concluons en énoncant les limites de notre recherche et des suggestions quant à des recherches ultérieures.

Mots-clés: Expo-sciences, motivation, questionnaire.

INCIDENCE OF STUDENTS' CHARACTERISTICS ON THEIR LEVEL OF MOTIVATION TO PARTICIPATE IN A SCIENCE FAIR

ABSTRACT: Science fairs are more and more popular among young pupils. The present study aims to identify the factors that can influence their motivation to participate in such events. To this end, we created a questionnaire intended to measure the degree of motivation of the participants in the Canada-Wide Science Fair. Our results indicate that participants are very motivated. However, and contrary to previous research, we also found that their degree of motivation is influenced by their age and grade. We conclude by acknowledging the limitations of our study and suggesting areas for subsequent research.

Keywords: Science fairs, motivation, questionnaire.

\section{INCIDÊNCIA DE CARACTERÍSTICAS DOS ESTUDANTES SOBRE SEU GRAU DE MOTIVAC̣ÃO PARA PARTICIPAR DE UMA EXPOSIC̣ÃO DE CIÊNCIAS}

RESUMO: As feiras de ciências são cada vez mais populares entre os jovens. Neste contexto, a motivação que possuem para participar deste tipo de atividade pode ser influenciada por vários fatores, os quais o presente estudo visa esclarecer. Para este fim foi elaborado um questionário para medir o grau de motivação dos participantes de uma feira nacional de ciências no Canadá, a qual é realizada anualmente e que reúne os melhores trabalhos do país. Nossos resultados indicam que os estudantes que responderam ao questionário são individuos altamente motivados. Entretanto, e ao contrário de algumas pesquisas anteriores, tal motivação é influenciada pela idade e nível de ensino dos respondentes. Outros fatores estudados não demonstraram influência significativa sobre a motivação dos participantes. Concluímos nosso estudo com uma abordagem dos seus limites e apresentamos sugestões para futuras pesquisas.

Palavras-chave: Feiras de ciências, questionário, motivação.
*University of Ottawa, Canadá. Email: Itrudel@uottawa.ca

** University of Ottawa, Faculty of education, Canadá. Email: greis@uottawa.ca

*** University of Ottawa, Faculty of education, Canadá. Email: Idionne@uottawa.ca 


\section{INTRODUCTION}

Chaque année des milliers de jeunes envahissent les salles des écoles, des écoles et des universités pour présenter à l'audience des projets à caractère scientifique. Le public est invité à venir écouter ces jeunes et à leur poser des questions concernant leur projet. Parmi ce public, certains font office de juges et c'est sur la base de leur verdict que les gagnants de cette exposition seront choisis. De telles activités, appelées expo-sciences, se déroulent à différentes échelles selon l'envergure de l'événement, pouvant regrouper quelques écoles à l'échelon régional jusqu'à plusieurs provinces à l'échelon national (Czerniak \& Lumpe, 1996). Des prix sont généralement accordés aux gagnants, qui peuvent consister en un montant d'argent ou en une opportunité de poursuivre des activités dans des centres de recherche.

Tout d'abord, les expo-sciences offrent une occasion pour les jeunes d'étudier un sujet, un problème ou un phénomène de leur environnement à l'aide de la démarche scientifique (Abernaty \& Lumpe, 2001). Les jeunes se mesurent alors aux difficultés d'un projet scientifique qu'ils ont eux-même choisi et poursuivent au meilleur de leurs connaissances et de leurs habiletés. La participation à un projet d'expo-sciences permettrait ainsi aux jeunes de développer de l'intérêt envers les sciences et favoriserait des attitudes favorables à celles-ci. À cet égard, un des aspects importants des expo-sciences concerne la plus grande autonomie dont dispose les jeunes qui s'engagent dans un projet d'expo-sciences. Cette autonomie semble reliée à ce que nous appelons une enquête scientifique authentique: choix du projet, conception d'une démarche permettant de résoudre le problème posé, présentation et analyse des résultats, discussion. Ainsi, l'expo-sciences constitue une opportunité pour les jeunes d'approfondir un sujet, occasion qui se manifeste plus rarement en milieu scolaire, où l'accent est mis davantage sur la couverture du curriculum. Il leur est alors possible de reprendre le même thème ou question de recherche pour le développer ou le raffiner au fur et à mesure de l'ajout de nouvelles informations, de discussions avec des pairs, leurs professeurs ou d'autres personnes de son environnement (oncle ingénieur, parent scientifique) ou la consultation de base de données (Trudel, Parent et Métioui, 2009.

Sur les plans personnels et sociaux, la participation à une expo-sciences peut être, pour les jeunes, une occasion de découverte de soi, leur permettant d'identifier leurs intérêts et de reconnaître leurs forces et leurs faiblesses. C'est aussi l'occasion de rencontrer d'autres jeunes qui partagent leurs intérêts, mais aussi des adultes qui peuvent les renseigner sur les perspectives d'études ou de carrières dans des domaines scientifiques et techniques (Guichard, 2007). Ainsi, la possibilité de faire des voyages, de rencontrer de nouvelles personnes correspond à une facette importante du développement de la personnalité et de la sociabilité des jeunes qui participent à une expo-sciences.

Malgré cet engouement et l'utilité manifeste pour le développement de l'intérêt et des habiletés scientifiques, les jeunes qui s'engagent dans un projet d'expo-sciences 
font face à plusieurs obstacles: manque de connaissances scientifiques pertinentes au projet, choix d'un thème trop difficile, manque de ressources matérielles pour réaliser les expériences nécessaires, manque de financement nécessaire à des déplacements éventuels, à l'achat de logiciels, etc., expertise insuffisante pour mener à bien le projet, compétition entre le temps alloué au projet et à d'autres intérêts (sorties, etc.) (Abernathy \& Vineyard, 2001). En outre, la participation des élèves aux expo-sciences fait souvent l'objet de critiques. Il leur est reproché leur caractère compétitif, qui peut générer de l'anxiété chez le jeune. En outre, ces compétitions sont souvent perçues comme réservées à l'élite des jeunes. La participation de ces derniers aux expo-sciences exigerait des fonds importants qui ne sont disponibles ou accessibles qu'aux couches favorisées de la société. Eu égard à ces considérations, notre recherche vise à évaluer l'influence des variables du milieu sur la motivation à participer des élèves du secondaire aux expo-sciences.

\section{CARACTÉRISTIQUES DES ÉLÈVES SUSCEPTIBLES D'INFLUENCER LEUR MOTIVATION À PARTICIPER À UNE EXPO-SCIENCE}

Notre revue de la littérature existante indique que les facteurs susceptibles d'influencer la motivation à participer aux expo-sciences sont : l'âge des participants, leur appartenance au genre masculin ou féminin, leur langue maternelle et seconde, leur niveau et rendement scolaire, l'occupation des parents et le caractère obligatoire ou libre de leur participation.

Concernant l'âge des participants, les études de Piaget ont montré que les habiletés cognitives se développent avec l'âge. Un degré de complexité plus élevé de la pensée permettrait au jeune de comprendre les aspects concrets et formels des phénomènes et des concepts impliqués dans son projet d'expo-sciences, ce qui pourrait l'amener à s'y engager et à le poursuivre (Byrnes, 2001). Par ailleurs, la curiosité envers le monde qui l'entoure et l'intérêt envers les sciences sont susceptibles de varier avec l'âge du jeune (Bell, 2003). Le genre masculin ou féminin constitue une dimension culturelle importante de la personnalité de l'élève et peut influencer la façon dont il s'engage dans des activités scientifiques. Dans le cadre de leur participation à une expo-sciences, garçons et filles seront amenés à entreprendre ou à utiliser les résultats d'expériences scientifiques et à en discuter les résultats, ce qui peut les inciter à s'engager dans ces activités d'une façon différente (Trudel, 1996). Ainsi, les garçons sont davantage intéressés dans la manipulation d'équipements et la construction de montages, alors que les filles préfèrent des activités d'observation des phénomènes et de traitement des données (Tobin et Gallagher, 1987). Dans les discussions à caractère scientifique, les filles recherchent davantage le consensus, alors que les garçons recherchent la confrontation et désirent gagner l'échange (Alexopoulou et Driver, 1997). À cet égard, il semble que les garçons apprécient davantage que les filles le caractère compétitif des expo-sciences (Abernathy \& Vineyard, 2001). En outre, 
des différences entre les genres se manifestent dans le choix des sujets, les filles préférant les thèmes biologiques alors que les garçons préfèrent choisir des thèmes de sciences physiques (Adamson, Foster, Roark \& Reed, 1998). Comme les jeunes sont susceptibles de s'engager dans ces différentes activités dans le cadre d'une expo-sciences, il est raisonnable de penser qu'il peut exister des différences entre garçons et filles dans leur motivation à y participer.

La langue peut avoir un impact sur l'apprentissage des sciences (Rivard, 2004). Cette affirmation est particulièrement importante eu égard à la réalité multilingue des pays influencés par un processus migratoire fort, comme le Canada. Ainsi, la familiarité avec le français ou anglais, qui sont les deux langues officielles du Canada et qui permettent de communiquer lors des expo-sciences, peut avoir une influence sur la difficulté qu'ils entrevoient à réaliser une telle tâche de nature scientifique. En outre, la langue est le véhicule de la pensée et ceci est d'autant plus vrai lorsqu'elle est utilisée pour transmettre l'information scientifique, l'organiser sous forme de tableaux, la présenter dans des graphiques, expliquer les concepts à d'autres et défendre son point de vue, élaborer des arguments pour convaincre.

Selon divers auteurs, les élèves se désintéressent de la science à l'école au fur et à mesure qu'ils suivent des cours de sciences (Bell, 2003). Qu'en est-il de la relation entre l'année scolaire et la motivation de l'élève à participer à une activité scientifique si celle-ci se déroule dans un cadre plutôt informel tel que les expo-sciences? Dans le même ordre d'idées, le rendement scolaire peut avoir une influence sur la motivation à apprendre (Foxx, 2001). En effet, les activités scientifiques sont abordées différemment selon que les rendements scolaires des élèves sont supérieurs ou inférieurs à la moyenne. Par exemple, les élèves plus faibles bénéficient davantage d'activités plus diversifiées, centrées sur les échanges oraux et l'établissement de liens entre la science et leur quotidien. À l'opposé, les élèves plus forts préféreraient davantage des activités où ils ont à développer des arguments scientifiques et à les expliquer à leurs collègues sous forme écrite (Rivard, 2004). Par conséquent, les jeunes, selon leur rendement scolaire, pourraient être motivés différemment à participer aux activités offertes dans le cadre des expo-sciences.

Guichard (2007) a démontré l'influence des contacts avec des adultes qui savent éveiller l'intérêt envers la science, que ce soit dans un cadre formel tel que l'école ou dans des activités scientifiques informelles comme les expo-sciences. Par exemple, dans un milieu où les parents occupent des emplois à nature scientifique, le jeune pourrait disposer de plus d'occasions de se mettre en contact avec la science, en visitant des musées de sciences, en regardant des émissions à caractère scientifique, etc. (McDonough, 1995). En outre, ces parents pourraient fournir au jeune des modèles à imiter. Dans cette optique, on peut se demander si l'influence du milieu familial, en particulier l'occupation scientifique ou non des parents, est susceptible de motiver davantage leur enfant à entreprendre un projet d'expo-sciences (Czerniak \& Lumpe, 1996).

Dans certaines écoles, la participation aux expo-sciences est obligatoire alors que dans certains cas, elle est laissée au choix de l'étudiant. Or, le caractère 
obligatoire de la participation à une expo-sciences semble aller à l'encontre de la marge de manœuvre laissée au jeune (qui constitue un des attraits d'un projet d'expo-sciences) et possiblement restreindre son intérêt envers le sujet choisi . Ce serait le cas, par exemple, si dans le cadre d'une participation obligatoire à l'expo-sciences, le jeune choisit son projet conformément aux désirs de son enseignant. Dans le cas où cette activité se déroule à l'école même, il est aussi possible que l'enseignant chapeaute l'activité et l'oriente conformément aux ressources disponibles à l'école. Néanmoins, le caractère obligatoire de la participation aux expo-sciences du jeune n'est pas nécessairement préjudiciable à son intérêt, tout au contraire, cet aspect est souvent nécessaire aux adolescents pour qu'ils s'engagent dans cette démarche, qu'ils n'ont peut-être jamais expérimenté (et comment peuton apprécier quelque chose que l'on ne connait pas?). Il est raisonnable de penser que certains jeunes peuvent par la suite découvrir de nouveaux intérêts et vouloir poursuivre dans ce domaine (Guichard, 2007). Dans la même veine, certains avancent que les jeunes n’ont peut-être pas acquis la maturité nécessaire pour faire eux-mêmes des choix éclairés et qu'un certain degré de contrainte est nécessaire pour les inciter à participer à une expo-sciences. Cette contrainte nécessaire leur permettrait de s'engager dans une activité qu'ils n'auraient peut-être pas choisi d'eux-mêmes mais qu'ils apprennent, une fois engagés, à aimer (Abernathy \& Lumpe, 2001). En conséquence, on peut alors se demander si le caractère obligatoire ou non de la participation de l'élève à l'expo-sciences a une incidence sur leur degré de motivation à participer à une expo-sciences.

\section{MÉTHODOLOGIE}

L'étude s'est déroulée dans le contexte d'une expo-sciences pancanadienne qui s'est déroulé à Ottawa en 2009. L'échantillon comprenait 94 participants de 12 à 18 ans, 52 filles et 42 garçons, provenant de diverses régions du Canada. Dans cette section, nous décrirons le choix de l'instrument de mesure de la motivation, la méthode d'analyse des réponses au questionnaire ainsi que la méthode de comparaison entre les groupes d'élèves possédant des caractéristiques différentes.

Le questionnaire soumis aux participants comportait 47 questions relativement à divers aspects de la motivation à participer aux expo-sciences. Ce questionnaire a été rédigé suite à une revue de littérature, où nous avons identifié les thèmes majeurs de la motivation à participer à une expo-sciences. La rédaction des questions s'est faite en adaptant les énoncés d'un questionnaire de motivation envers les sciences que nous avons adapté au contexte des expo-sciences (Tuan, Chin \& Shieh, 2005). Les étapes de la validation de ce questionnaire sont décrites dans Dionne, Reis, Trudel, Guillet, Kleine et Hancianu (2011). Une fois ces diverses étapes complétées, nous avons rédigé plusieurs formes de ce questionnaire adapté aux différents regroupements des jeunes participants à l'expo-sciences : français 
ou anglais, juniors ou séniors. Ceci nous donne quatre versions : français junior, français sénior, anglais junior, anglais sénior.

Outre la langue de rédaction des questions (français ou anglais), la principale différence en ce qui concerne les différents formats des questionnaires concerne l'année scolaire des jeunes auquel le questionnaire est destiné, le questionnaire junior étant destiné aux jeunes de niveaux scolaires de 7 à à la $10^{\text {ème }}$ année alors que le questionnaire sénior était destiné aux niveaux scolaires allant de

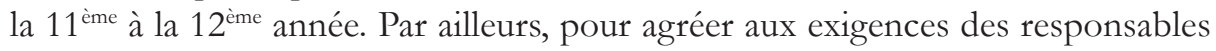
de l'expo-science, nous avons utilisé des échelles différentes pour les juniors et les séniors. À cet égard, l'échelle pour les juniors comprenait trois niveaux : en désaccord, sans opinion, en accord. L'échelle pour les séniors comprenait les cinq niveaux suivants : très en désaccord, en désaccord, sans opinion, en accord, très en accord. Nous incluons en annexe la version destinée aux élèves des années 7 à 10 (junior). Les autres versions sont sensiblement les mêmes à l'exception de la structure de l'échelle qui compte 5 niveaux plutôt que trois.

Selon Oppenheim (1992), les avantages d'un questionnaire utilisant une échelle de Likert sont les suivants : 1) le questionnaire peut inclure un grand nombre d'énoncés pouvant être répondu en un cours laps de temps; 2) le questionnaire peut fournir des renseignements précis sur le degré d'accord ou de désaccord relativement a chacun des énoncés; 3) les résultats obtenus peuvent être d'un haut degré de fidélité; 4) les énoncés peuvent être formulés facilement. Cependant, certains critiquent l'utilisation de méthodes quantitatives dans les études sur la motivation et les attitudes envers les sciences (Oppenheim, 1992). Ainsi, les méthodes quantitatives seraient utiles pour identifier la nature d'un problème, mais elles seraient peu efficaces pour comprendre en profondeur celui-ci. En outre, le format «papier et crayon » sous lequel on retrouve la majorité des questionnaires, est limité quant à la quantité de renseignements qu'il peut produire. En effet, ce format permet seulement une gamme étroite de réponses et ne fournit pas l'occasion d'explorer la pensée du répondant. En outre, peut-être de façon plus significative, ces instruments sont construits selon la perspective des chercheurs (Oppenheim, 1992).

En dépit de ces restrictions, l'échelle de Likert est utile pour diviser les gens en groupes selon leur motivation ou leur attitude envers les sciences et permet au chercheur d'établir un diagnostic sommaire de leur motivation envers les expo-sciences (Oppenheim, 1992). De plus, il faut se rappeler que cette sorte d'échelle ne produit pas de score absolu sur une variable donnée, mais plutôt une comparaison entre le degré de motivation du répondant et la difficulté à être en accord ou en désaccord avec les énoncés du questionnaire. Pour améliorer la fidélité de l'échelle, il est préférable de constituer un ensemble d'énoncés apparentés pour qu'une direction de motivation croissante puisse être établie en réduisant l'erreur de mesure (Wright \& Masters, 1982; Oppenheim, 1992). Bien que la validation de l'échelle puisse être faite avec la théorie classique de la mesure, la tendance actuelle est d'analyser les patrons de réponses obtenus à 
l'aide de la théorie de la réponse à l'item. À la différence des modèles classiques qui établissent d'après un mode " ad hoc » les qualités psychométriques du questionnaire, les modèles de réponse à l'item contiennent des procédures internes qui permettent d'établir ces qualités (Bond \& Fox, 2007).

Comme, d'après nos analyses, le type d'échelle utilisé n'a pas influencé de façon notable le degré de motivation à participer à une expo-sciences, nous avons choisi, pour améliorer la précision de nos résultats, de modifier l'échelle à 5 niveaux en combinant les niveaux « très en désaccord » et « en désaccord », et « très en accord » et « en accord ». Une telle modification nous permet d'utiliser une échelle unique à trois niveaux pour analyser les résultats des juniors et des séniors combinés (Bond et Fox, 2007). Afin de déterminer si les énoncés peuvent être ordonnés suivent une échelle hiérarchique et de calibrer celle-ci, nous avons choisi le modèle d'analyse des échelles d'Andrich (Bond et Fox, 2007). Ce modèle donne la probabilité « Pnik » qu'un élève d'un degré de motivation donné « $\beta_{n}$ » choisisse une des catégories allant du désaccord à l'accord dont le degré de difficulté spécifique est « $\delta_{\mathrm{ik}}$ ». Le calcul s'obtient avec l'équation du modèle d'Andrich (Wright et Masters, 1982 ; Bond et Fox, 2007):

$$
\mathrm{P}_{\text {nik }}=\exp \left(\beta_{\mathrm{n}}-\delta \mathrm{ik}\right) /\left[1+\exp \left(\beta_{\mathrm{n}}-\delta_{\mathrm{ik}}\right)\right]
$$

Où $\quad \beta_{\mathrm{n}}$ est l'habileté de l'élève $\mathrm{n}$ en logits

$\delta_{\mathrm{ik}}=\left(\delta i+\tau_{\mathrm{k}}\right)$ où di est la difficulté de l'item i en logits et

tk est la mesure du seuil de réponse associé à chacun des sauts de compréhension « $\mathrm{k} »$.

Le double indice dans l'expression $\delta_{\mathrm{ik}}$ est nécessaire ici parce que le modèle d'Andrich sépare le degré de difficulté $\delta_{i k}$ associé à chaque question en deux composantes: une sur la difficulté de la question dans son ensemble et l'autre sur la difficulté de chaque saut $\tau_{k}(k=1,2,3)$ entre les réponses possibles aux questions. Dans le modèle de l'échelle d'appréciation, la valeur du paramètre associée à chaque saut, « $\tau_{\mathrm{k}}$ », ne varie pas d'une question à l'autre, seule la difficulté de la question dans son ensemble varie d'une question à l'autre.

Àpartir du patron de réponses de l'élève, il est possible de situer son degré de motivation sur une échelle pourvu que les différents énoncés puissent être ordonnés suivant une ligne croissante de difficulté, c'est-à-dire que le test soit unidimensionnel. Le test est unidimensionnel si la valeur moyenne pondérée des résidus standardisés (ajustement pondéré) est comprise dans un intervalle de confiance de deux écarts-types au seuil alpha $=0,05$. Lorsque l'ajustement pondéré est supérieur à +2 , ceci implique que les variations dans la mesure de la motivation sont supérieures aux attentes du modèle. Ce serait le cas d'un élève qui est en accord avec des énoncés de question plus difficiles que son degré de motivation. Dans le cas où l'ajustement pondéré est inférieur à -2 , cela signifie que ces variations sont inférieures à ce que permet le modèle (Bond et Fox, 2007). 
L'utilisation du modèle de l'échelle d'appréciation nous permet de répartir les divers degrés de motivation mesurés selon une échelle ayant les propriétés d'intervalle. Comme notre objectif de recherche s'intéresse à l'incidence des caractéristiques des élèves sur leur degré de motivation à participer à une expo-science, les propriétés d'intervalle de l'échelle ainsi constitué nous permettent d'utiliser les statistiques paramétriques, en particulier l'analyse de la variance afin de déterminer si par exemple, il existe un différence significative entre les différents niveaux des variables indépendantes (i.e. le genre, le niveau scolaire, l'âge, etc.) et la variable dépendante (le degré de motivation).

\section{ANALYSE DES RÉSULTATS}

Pour obtenir les propriétés du questionnaire, nous avons soumis les résultats de notre test à l'analyse du logiciel RUMM 2030. Ce logiciel analyse les réponses des élèves selon le modèle de l'échelle d'appréciation. D’après nos résultats, nous constatons tout d'abord que seuls deux énoncés (3 et 38) démontrent un ajustement pondéré supérieur à +2 ou inférieure à -2 , ce qui se traduit par un ajustement déficient au modèle de l'échelle d'appréciation choisi. Néanmoins, à la lumière des caractérististiques homogènes de la population considérée, nous avons décidé de conserver ces énoncés à des fins de validation ultérieure avec un échantillon de répondants plus diversifié.

En ce qui concerne les mesures de tendance centrale, nos résultats indiquent que les énoncés se répartissent autour d'une moyenne de zéro (par convention) avec un écart-type de 2.27 logits. Quant aux élèves, ils se répartissent autour d'une moyenne de 1.81 logit avec un écart-type de 0.44 logit. Par conséquent, le degré moyen de motivation des répondants est supérieur au degré de difficulté des énoncés. En outre, la variation des degrés de motivation des répondants est de beaucoup inférieure à la variation des degrés de difficulté des énoncés. Par conséquent, les résultats indiquent que les répondants sont motivés et qu'ils constituent un échantillon plutôt homogène quant à celle-ci. Par ailleurs, en étudiant les valeurs des degrés de difficultés des énoncés, nous constatons que la majorité des mesures de ces degrés sont regroupées dans une fourchette allant de -2,23 logits à 3,41 logits, ce qui dénote que les énoncés du questionnaire couvre un large intervalle des degrés de motivation.

Pour déterminer si les énoncés sont suffisamment répartis en niveaux distincts pour définir une ligne d'intensité croissante de la motivation, nous avons déterminé le degré de séparation de ceux-ci : 5.11. Ceci signifie que l'écart-type ajusté des degrés de difficulté est au moins cinq fois plus grand que la racine carrée de la moyenne des carrés des erreurs de calibration. Ce résultat indique un bon degré de séparation (Wright et Masters, 1982). La fidélité avec lequel l'échantillon sépare les items est donnée par RUMM 2030, soit 0,96. Par conséquent, nous concluons que les énoncés du questionnaire sont 
suffisamment séparés pour définir une intensité croissante de la motivation et que cette séparation est très fidèle.

Le nombre de groupes distincts dans lesquels se répartissent les items est 7,1. Par conséquent, les énoncés du questionnaire se répartissent en 7 groupes distincts selon une ligne d'intensité croissante avec une excellente fidélité. En outre, les élèves se répartissent deux groupes distincts quant au degré de motivation (ceux qui sont motivés et ceux qui ne le sont pas).

Puisque les personnes et les énoncés sont mesurés à l'aide d'une échelle commune, il est intéressant de comparer la répartition de motivation des répondants et les degrés de difficulté des énoncés du questionnaire.

Nous présentons, à la figure 1, les distributions comparées des habiletés des élèves et des degrés de difficulté des super-items. Il est à noter que les éléments ayant des valeurs extrêmes ne sont pas placés selon leur mesure, mais sont placés aux extrémités supérieures ou inférieures selon le cas dans la colonne appartenant à leur facette. Par ailleurs, le «+ » ou le « - » indiquent que les mesures de la facette sont orientées positivement ou négativement. Enfin, dans la colonne de compréhension de l'élève, le symbole «*» représente la valeur « 2 » et le symbole « $\bullet$ représente la valeur « 1 ».

La figure 1 nous indique que la majorité des degrés de motivation des répondants se situe au dessus de la valeur moyenne des degrés de difficulté des énoncés. En outre, la distribution des degrés de motivation des élèves est étroite comparée à celle des degrés de difficulté des énoncés. On peut en conclure que l'échantillon choisi est homogène relativement à leur motivation à participer à une expo-science et qu'ils sont motivés à le faire. Enfin, l'étude de la figure 1 nous permet d'apprécier à quel point l'échantillon de nos élèves est adéquat pour obtenir une bonne mesure du degré de difficulté des super-items et des niveaux de compréhension. En effet, idéalement, les distributions des élèves et des degrés de difficulté devraient se recouper pour qu'il soit possible d'obtenir de l'information sur la difficulté des super-items et sur la mesure des habiletés des élèves (Wright et Stone, 1979). 
Figure 1: Distributions comparées des degrés de motivation des élèves et des degrés de difficulté des items

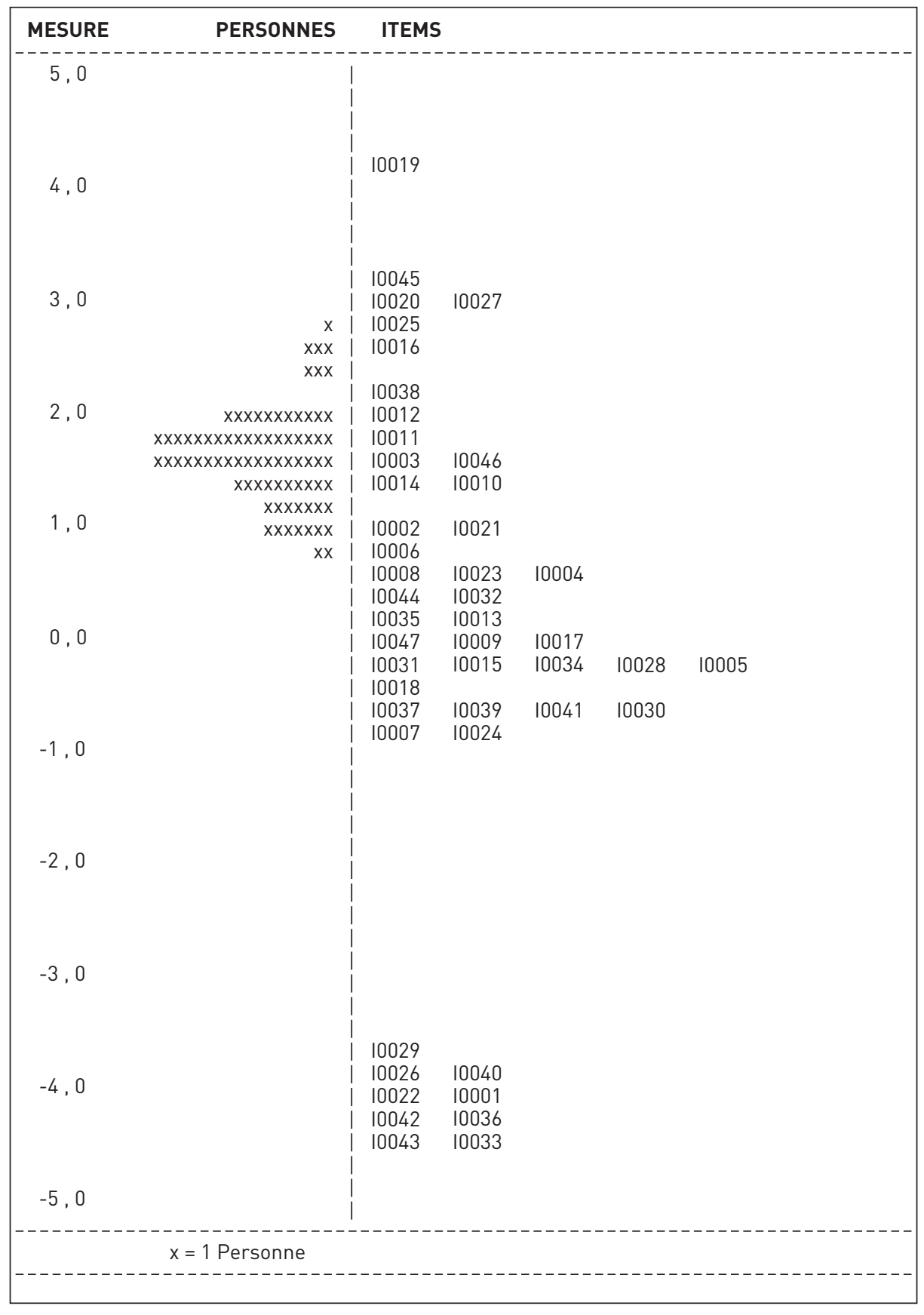

Quant aux catégories, le tableau 1 nous renseigne sur les choix des élèves dans chaque catégorie. D'après le tableau 1, nous nous apercevons que pour 
l'ensemble des items du test, les réponses aux questions selon les catégories 1 (en désaccord) et 2 (aucune opinion) sont choisies respectivement par 19\% et 15\% des répondants alors que la catégorie 3 (en accord) est choisie par 66\% des élèves. Ceci est en accord avec notre constat que ce sont des élèves très motivés et qu'ils forment relativement à ce questionnaire de motivation une population assez homogène.

Tableau 1: Description des caractéristiques des diverses catégories de réponse

\begin{tabular}{|l|l|l|l|l|l|l|}
\hline Score & $\begin{array}{l}\text { Fréquence } \\
\text { absolue }\end{array}$ & $\begin{array}{l}\text { Fréquence } \\
\text { relative } \\
(\%)\end{array}$ & $\begin{array}{l}\text { Fréquence } \\
\text { cumulée } \\
(\%)\end{array}$ & Saut & $\begin{array}{l}\text { Difficulté } \\
\text { du saut } \\
\text { (logit) }\end{array}$ & $\begin{array}{l}\text { Erreur- } \\
\text { type } \\
\text { (logit) }\end{array}$ \\
\hline 1 & 835 & 19 & 19 & & & \\
\hline 2 & 656 & 15 & 34 & 1 & 0.18 & .05 \\
\hline 3 & 2901 & 66 & 100 & 2 & -0.18 & .04 \\
\hline
\end{tabular}

Pour chacune des caractéristiques mentionnées à la section 2, nous allons maintenant présenter d'une part les statistiques descriptives de notre échantillon et d'autre part les résultats des analyses de la variance permettant de déterminer si l'effet de ce facteur sur la motivation est signification au seuil alpha de 0.05 (seuls les résultats significatifs seront présentés en tableau pour ne pas alourdir la présentation). On voit ici (Figure 2) que parmi les participants, les catégories d'âge les plus fréquentées sont celle de 16 ans (27\% de l'ensemble des participants) et de 13 ans (22\%).

Figure 2: Proportion en \% des élèves en selon les catégories d'âge

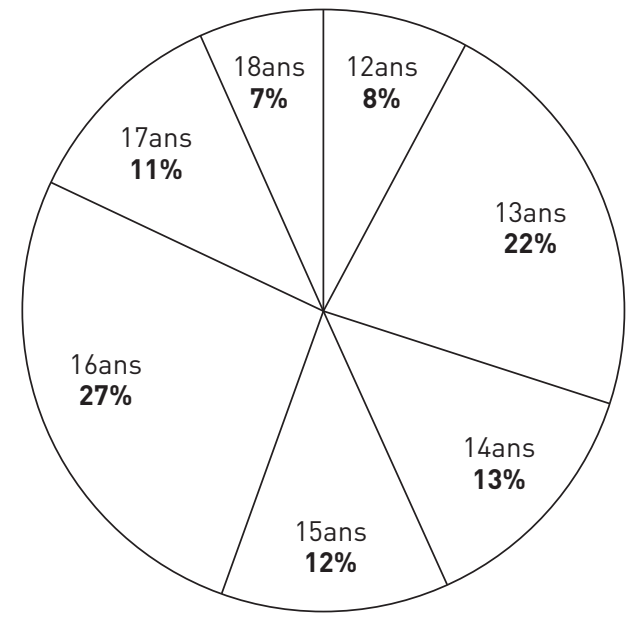

Suivent dans l'ordre celles et avec des proportions voisines celles de 14 ans $(13 \%)$, de 15 ans $(12 \%)$, de 17 ans (11\%), de 12 ans (8\%), et de 18 ans (7\%). 
Par conséquent, concernant leur participation aux expo-sciences, on constate deux pics l'un à 16 ans (27\%) et l'autre à 13 ans (22\%) (voir figure 2).

Quant à leur degré de motivation, il semble que l'on observe deux sommets également, l'un à quatorze ans et l'autre à 17 et 18 ans (voir figure 3).

Figure 3: Degré de motivation en fonction de l'âge des participants

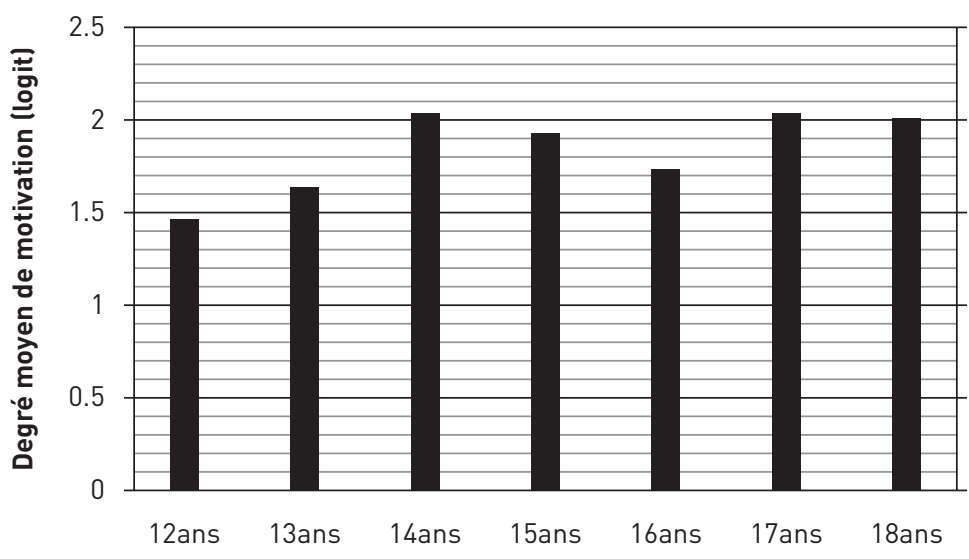

Âge du participant

On constate que ces sommets de motivation ne correspondent pas aux pourcentages les plus élevés de la participation (voir fig. 2), mais qu'ils suivent ces derniers d'une année. Ainsi, un des sommets de la motivation (14 ans) suit d'une année l'un des sommets dans la participation (13 ans).

L'analyse de la variance montre qu'au moins un des résultats de motivation obtenu se distingue de façon statistiquement significative des autres au seuil alpha de $0,05(\mathrm{p}=0,007<0,05)$ (voir tableau 2).

Tableau 2: Analyse de la variance du degré de motivation en fonction des catégories d'âge des participants

\begin{tabular}{|l|l|l|l|l|l|}
\hline $\begin{array}{l}\text { Source de la } \\
\text { variation }\end{array}$ & $\begin{array}{l}\text { Somme des } \\
\text { carrés }\end{array}$ & $\begin{array}{l}\text { Degrés de } \\
\text { liberté }\end{array}$ & $\begin{array}{l}\text { Somme } \\
\text { des carrés } \\
\text { moyens }\end{array}$ & Statistique F & Probabilité \\
\hline $\begin{array}{l}\text { Entre les } \\
\text { catégories }\end{array}$ & 3,23 & 6 & 0,54 & 3,187134 & 0,007275 \\
\hline $\begin{array}{l}\text { À l'intérieur } \\
\text { des } \\
\text { catégories }\end{array}$ & 14,01 & 83 & 0,17 & & \\
\hline Total & 17,24 & 89 & & & \\
\hline
\end{tabular}

En ce qui concerne l'année scolaire (voir figure 4), la participation des jeunes à l'expo-sciences est la plus importante pour les années $8(22 \%)$ et 11 (24\%). 
Figure 4: Degré de motivation des participants en fonction de l'année scolaire

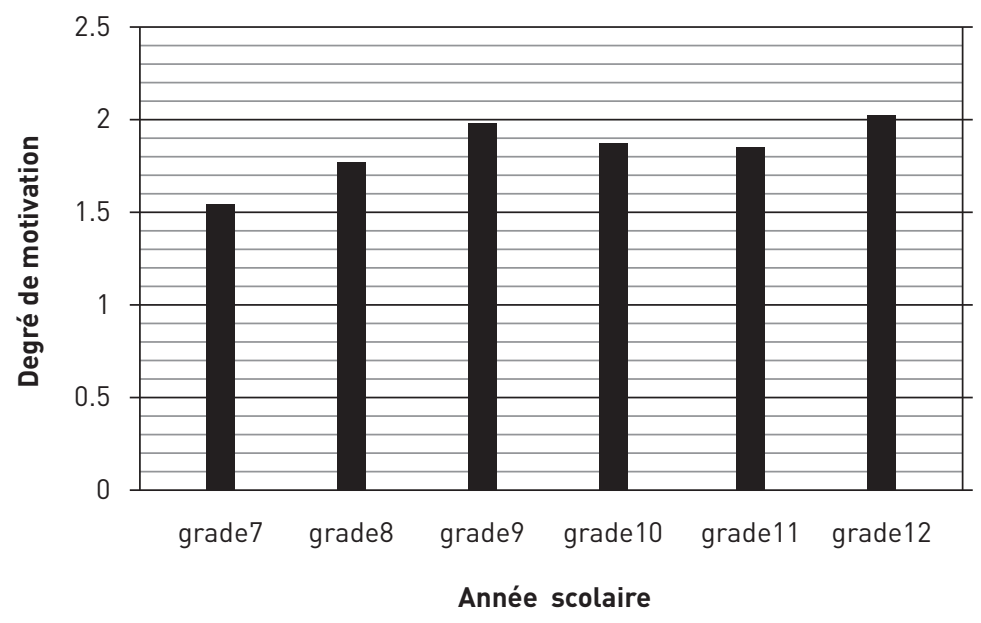

Suivent des participations presqu'équivalentes pour les niveaux 7, 9, 10 et 12 (voir figure 4). Comme les résultats en ce qui concerne l'influence de l'année scolaire sur la motivation sont similaires à ceux de l'âge, nous allons brièvement en décrire les points essentiels.

Relativement au degré de motivation, on constate deux valeurs plus élevées de motivation aux niveaux $9(1,983)$ et $12(2,018)$ que les autres niveaux qui ont des degrés de motivation presqu'équivalents (entre les niveaux 8, 10 et 11, la motivation varie entre 1,752 et 1,860$)$. Suit la motivation du niveau 7 qui est la plus basse soit de 1,435 (voir figure 4).

D’une façon similaire aux résultats obtenus en ce qui concerne l'âge, l'analyse de la variance montre que les différences constatées dans la motivation entre les années scolaires sont significatives au seuil alpha de 0.05 ( $p<0.015)$. Ces résultats sont intéressants en ce sens qu'ils vont à l'encontre de la croissance du désintérêt envers les sciences au fur et à mesure que les élèves progressent dans le cursus des sciences du secondaire. Cette croissance n'est pas constante toutefois, elle se manifeste par des montées suivies de descente et de remontées au cours du cheminement scolaire de l'élève. L'apogée de la première montée se situe près de la fin du premier cycle du secondaire (soit en $9^{\text {ème }}$ année) et la seconde à la fin du deuxième cycle (soit en $12^{\text {ème }}$ année). Fait curieux, les deux pics de motivation suivent d'une année, les années de plus grande participation à l'expo-sciences (soit $8^{\text {ème }}$ année et $11^{\mathrm{ème}}$ année respectivement).

On peut ici se demander compte tenu de la similarité des courbes de la motivation en fonction de l'âge et du niveau scolaire, si les effets observés ne sont pas reliés au même facteur, soit le développement cognitif qui s'observe à cet âge (Byrnes, 2001). Par ailleurs, ce décalage entre la participation et la motivation peut donner des arguments à ceux qui affirment qu'il est important de mettre en contact les jeunes avec la science plutôt que d'attendre qu'ils s'engagent d'eux-mêmes. 
En ce qui concerne la langue maternelle, nos résultats indiquent qu'il y a 49 participants (54\%) dont la langue maternelle est l'anglais, 24 (27\%) le français, 7 $(8 \%)$ parlent une autre langue que le français ou l'anglais. Par ailleurs, 10 participants $(11 \%)$ n'ont pas répondu à cette question. Ces pourcentages sont sensiblement les mêmes que la population canadienne dont $57 \%$ ont indiqué que l'anglais est leur langue maternelle et 22\% le français (Statistique Canada, 2006a).

Quant à leur degré de motivation, ceux dont la langue maternelle est l'anglais ont des degrés de motivation inférieurs aux autres $(1,708)$ alors que ceux qui parlent le français ou une autre langue ont un degré supérieur et sont similaires entre elles (1,904 soit 1,946). Par ailleurs, 10 participants n'ont pas répondu à cette question et leur degré de motivation est le plus élevé (1,991). Toutefois, aucune différence entre les catégories recensées n'est significative $(p=0,106>0,05)$ au seuil alpha considéré (alpha $=0,05)$.

En ce qui concerne l'occupation scientifique ou non des parents, les participants se répartissent pratiquement également entre ceux dont les parents ont une occupation scientifique $(42 \%)$ de ceux qui n'en ont pas (47\%). Relativement au degré de motivation, nous observons un léger avantage au niveau de la motivation des participants pour ceux dont l'un des parents (père, mère, frère ou sœur) occupe un emploi scientifique $(1,802)$ comparé à ceux dont aucun des parents n'en occupe $(1,775)$ mais cette différence n'est pas significative $(p=0,38$ $>0,05)$ au seuil alpha considéré $(0,05)$.

Il est important toutefois de remarquer que la proportion de jeunes des expo-sciences dont un des parents occupe un emploi à caractère scientifique ou technique excède largement la proportion des emplois scientifiques dans la population en général (5.6\% d'après Statistique Canada, 2006a) même si elle n'engendre pas un degré de motivation significativement plus grand chez les premiers.

En ce qui concerne le caractère obligatoire ou non de la participation à une expo-sciences le pourcentage de participants ayant choisi de participer $(45 \%)$ est légèrement supérieur au pourcentage dont la participation était obligatoire (40\%). Quant au degré de motivation, nous observons un léger avantage au niveau de la motivation des participants qui ont choisi (1.6) relativement à celle dont les participants étaient obligés (1.46) mais cette différence n'est pas significative $(\mathrm{p}=0,21>0,05)$ au seuil alpha considéré $(0,05)$.

Nous avons également étudié l'incidence des performances scolaires sur le degré de motivation des participants. À cette fin, la question posée demandait au participant d'évaluer selon son jugement sa performance selon les catégories suivantes : les 10\% moins performants de la classe, en dessous de la moyenne, la moyenne, au dessus de la moyenne, meilleur 10\% de la classe. Les résultats sont indiqués au tableau 3. Selon la troisième colonne du tableau 3, on constate que la grande majorité des participants se classe dans les catégories supérieures de la performance et qu'aucun ne juge sa performance inférieure à la moyenne. Quant à la motivation, son degré ne varie que très peu selon la performance, allant 1.549 pour une performance moyenne à 1.631 pour une performance au 
dessus de la moyenne, alors que la motivation des 5\% plus performants est de 1.575. Sans surprise, ces différences ne sont pas significatives $(p<0.997)$ au seuil alpha considéré (0.05).

Tableau 3: Valeur moyenne de la motivation des participants selon leur performance scolaire

\begin{tabular}{|l|l|l|l|l|}
\hline Niveau & Description & Fréquence & Moyenne & Écart-type \\
\hline 1 & Les moins & 0 & 0.000 & 0.00 \\
\hline 2 & Sous moy. & 0 & 0.000 & 0.00 \\
\hline 3 & Moyenne & 6 & 1.549 & 0.29 \\
\hline 4 & Dessus moy. & 19 & 1.631 & 0.40 \\
\hline 5 & Les plus & 64 & 1.575 & 0.45 \\
\hline 6 & Manquante & 5 & 1.538 & 0.51 \\
\hline
\end{tabular}

Quant au genre masculin et féminin (tableau 4), les femmes sont légèrement plus nombreuses que les hommes (52 relativement à 42) et leur motivation légèrement supérieure (1.633 relativement à 1.520$)$. Néanmoins, cette différence n'est pas significative $(\mathrm{p}<0.21)$ au seuil alpha considéré $(0.05)$.

Tableau 4: Valeur moyenne de la motivation des participants selon leur genre

\begin{tabular}{|l|l|l|l|}
\hline Genre & Fréquence & Moyenne & Écart-type \\
\hline Masculin & 42 & 1.520 & 0.39 \\
\hline Féminin & 52 & 1.633 & 0.46 \\
\hline
\end{tabular}

\section{DISCUSSION}

Concernant la participation des élèves aux expo-sciences, un aspect moins étudié concerne l'influence des facteurs du milieu tels que l'âge, le genre, le niveau scolaire, l'occupation scientifique ou non des parents, les langues maternelles et secondes, la performance scolaire et le caractère obligatoire ou libre deleur participation.

Tout d'abord un premier constat nous vient de l'étude des réponses des participants au questionnaire dans son ensemble. Nous avons observé que le degré de motivation des élèves était supérieur au degré de difficulté moyen des énoncés du questionnaire de sorte qu'en moyenne les participants affichent un degré d'accord élevé avec la plupart des énoncés. En outre, l'écart-type des degrés de motivation des élèves est inférieur à l'écart-type des degrés de difficulté des énoncés. De ces constats se dégage un portrait de l'ensemble des participants affichant un degré élevé 
de motivation et une distribution plutôt homogène dans les résultats. À l'inverse, les degrés de difficulté des énoncés couvrent un large éventail de l'échelle logit, ce qui en fait un instrument de diagnostic approprié de la motivation. Toutefois, il serait approprié de poursuivre la validiation de ce questionnaire avec des échantillons d'élèves ayant des degrés de motivation plus variées (i.e. qui sont peu ou pas motivés à participer à une expo-sciences) pour accroître la précision des mesures surout aux extrémités de l'échelle et la fidélité de la mesure en général.

En ce qui concerne l'incidence des facteurs mentionnés précédemment sur la motivation, nous constatons en premier lieu que la motivation des élèves à participer à une expo-sciences s'accroit de façon significative avec l'âge et avec l'année scolaire. Compte tenu du nombre restreint de participants (94), il n'a pas été possible d'étudier l'effet de ces deux facteurs séparément. Néanmoins, ce résultat va à l'encontre des résultats des recherches précédentes qui déplorent le désintérêt progressif des élèves envers les sciences au fur et à mesure qu'ils progressent dans le cursus scolaire (Bell, 2003). De même, ils contredisent les résultats d'Abernathy et Lumpe (2001) qui avaient observé une différence dans la participation aux expo-sciences entre le début et la fin du secondaire. Il faut noter que l'échantillon de cette dernière étude était beaucoup plus important et davantage diversifié que le nôtre. À cet égard, il est possible que les relations entre la motivation et l'âge ou l'année scolaire soient différentes pour notre échantillon d'élèves dans l'ensemble très motivés, dont les performances sont supérieures à la moyenne. Malgré tout, on peut aussi avancer que le caractère informel des exposciences ait quelque chose à voir avec cet intérêt envers ce type d'activités. En effet, comme le soulignons précédement, les élèves participants à une expo-sciences ont l'occasion de s'engager dans un sujet qui les intéresse, disposent aussi de plus d'autonomie pour la poursuite de leur projet que dans le cadre plus formel de l'école et peuvent tirer des souces de motivation dans les interactions avec des adultes plus connaissants à même de les guider (Dionne et al., 2011).

En ce qui concerne ces deux facteurs, un détail inexpliqué concerne la relation entre la participation maximale et le degré de motivation le plus élevé. Ce dernier arrive en effet l'année suivant le sommet dans la participation. On peut se demander si conformément à ce résultat la participation à une expo-sciences précède le développement de la motivation. Pour confirmer cette hypothèse, un devis longitudinal permettrait de suivre les élèves au cours des années. À cet égard, il serait également intéressant d'étudier la motivation d'élèves ayant participé à plusieurs expo-sciences.

Le rôle de la langue a été maintes fois souligné dans l'apprentissage des sciences (Rivard, 2004). Or, nos résultats vont à l'encontre de ces constats, ce qui est d'autant plus surprenant que le type d'activités incluses dans une expo-sciences comprend une large part de discussion, de communications, d'échanges, où l'usage de la langue joue un rôle important.

Contrairement à une opinion commune reprise par divers auteurs, il semble que l'occupation scientifique ou non des parents n'ait pas d'incidence 
significative sur le degré de motivation des parents. Ceci pourrait s'expliquer peut-être par les efforts que les jeunes ont à faire pour se rendre à l'expo-sciences, efforts qui pourraient être facilités par un environnement favorable aux sciences. Par contre, ceux qui ne disposent pas d'un tel environnement doivent redoubler d'effort et donc être très motivés, ce qui semble le cas de nos participants dont le degré de motivation est élevé. Malgré tout, si on compare le pourcentage de parents qui occupent des emplois scientifiques dans notre échantillon (42\%) et celui de la population en général (5.6\%) (Statistique Canada, 2006a), il y a lieu de se demander si l'occupation scientifique des parents n'aurait pas une influence sur le pourcentage de participation des élèves à une expo-sciences (McDonough, 1995). On pourrait certes faire l'hypothèse qu'un milieu familial favorable aux sciences serait plus susceptible à encourager le choix de l'élève à s'engager dans une expo-sciences. Seules des recherches subséquentes pourront répondre à cette question.

La présence en nombre équivalent de garçons et de filles montre que ces dernières occupent maintenant une place important dans les loisirs scientifiques, contrairement à la situation de l'emploi dans les secteurs techniques et scientifiques où il existe un déséquilibre entre le pourcentage d'emplois occupés par les femmes et les hommes, déséquilibre à l'avantage de ces derniers (Statistique Canada, 2007). Par ailleurs, il n'existe pas de différence significative entre leur degré de motivation et celles de leurs congénères masculins, conformément aux résultats d'Abernathy et Vineyard (2001). Quant au dernier facteur, la performance scolaire, il ne semble pas influencer de façon significative les résultats, contrairement à une opinion répandu que les expo-sciences sont réservés aux élèves les plus doués.

\section{CONCLUSION}

L'étude d'une population telle que les élèves participant à une expo-science d'envergure nationale est intéressante à considérer puisqu'il s'agit d'élèves ayant réussi à se qualifier au plus haut niveau en réusissant à toutes les étapes des compétitions locales, régionales, provinciales et nationales. En général, les études portent sur leurs caractéristiques propres telles que leur sentiment d'auto-efficacité (Dionne et al., 2011), leurs habiletés scientifiques, etc. On espère ainsi identifier les caractéristiques les plus susceptibles à favoriser l'intérêt des jeunes envers les sciences. De telles informations nous offriraient des pistes pour rénover l'enseignement des sciences au niveau secondaire et surtout d'améliorer l'image que les jeunes se font des sciences.

Malgré tout, les caractéristiques particulières de cet échantillon constitué d'élèves motivés par les sciences, dont les performances scolaires sont supérieures à la moyenne, limitent la possibilité de généraliser nos résultats à l'ensemble de la population. Par aileurs le nombre limité de participants ne nous a pas permis de vérifier certaines hypothèses spécifiques concernant les interactions possibles entre les facteurs, tel que l'âge et l'année scolaire, sur la participation. Enfin, même 
si l'utilisation d'un questionnaire est adéquate pour décrire la participation des élèves aux expo-sciences et évaluer l'incidence de leurs caractéristiques sur leur motivation, elle ne permet pas d'approfondir l'analyse en identifiant les raisons des effets observés.

Ces résultats néanmoins sont intéressants en ce qu’ils contredisent certaines opinions reçues concernant les participants aux expo-sciences, tels le caractère obligatoire de la participation qui serait selon certaines préjudiciable au développement de la motivation chez le jeune, ou que les jeunes dont les parents occupent un emploi scientifique seraient privilégiés. Pour vérifier sur une plus large échelle ces résultats, nous espérons poursuivre cette recheche avec des échantillons plus diversifiés, notamment composé d'élève ayant des degrés de motivation plus variés.

\section{RÉFÉRENCES}

ABERNATHY, T.V; VINEYARD, R.N. Academic competitions in science: What are the rewards for students. The Clearing House, 74(5), 269-276, 2001.

ADAMSON, L.B. et. al (1998). Doing a science project: Gender Differences during childhood. Journal of Research in Science Teaching, [s.1], 35(8), 845-857.

ALEXOPOULOU, Evinella ; ROSALIND, Driver. «Gender differences in small group discussion in physics». International Journal of Science Education, 19(4),

393-406, 1997.

BELL, Karine. (2003). Analyse transversale de l'évolution de la motivation et des attitudes des élèves de première à cinquième secondaire; Mémoire de maitrise. Montréal : Université du Québec à Montréal,2003.

BOND, T.G; FOX, C.M. (2007). Applying the Rasch model: Fundamental measurement in the human science. 2nd ed. Mahwah (NJ): Lawrence Erlbaum Associates, 2007.

BYRNES, James P. Cognitive development and learning in instructional contexts. Boston : Allyn and Bacon, 2001.

CZERNIAK, C.M.; LUMPE, A.T. Predictors of science fair participation using the theory of planned behavior. School Science and Mathematics, [s.1], 96(7), 355-361, 1996.

DIONNE, L. et. al .Students' sources of motivation for participating in science fairs: an exploration study within the Canada-wide science fair 2008. International Journal of Science and Mathematics Education, [s.l], 2011.

FOXX, R.E. (2001). Evaluation of a constructivist pedagogy: Influence on critical thinking skills, science fair participation and level of performance. Dissertation Abstract International, [s.I], 62(02A), 05162001.

Guichard, F. Comment devient-on scientifique? Enquête sur la naissance d'une vocation. Les Ulis (France) : EDP Sciences, 2007.

MCDONOUGH, S.G. How parental support affects students' attitudes toward the science fair. Report: ED390707, 1995.

OPPENHEIM, A.N. Questionnaire design, interviewing and attitude measurement. London (UK): Pinter Publishers, 1992.

Rivard, L.P. (2004). Are language-based activities in science effective for all students, including low achievers? Science education, [s.l], 88 (3). 420-442.

Statistique Canada. (2006a). The Daily, 11 May 2006, disponible sur le site : <http://www.statcan. gc.ca/daily-quotidien/060511/dq060511b-eng.htm>

Statistique Canada. (2006b). Recensement de la population canadienne 2006, disponible sur le site : <http://www.statcan.gc.ca/tables-tableaux/sum-som/101/cst01/demo11a-eng.htm>

Statistique Canada. (2007). The Daily, 24 October 2007, disponible sur le site:

<http://www.statcan.gc.ca/daily-quotidien/071024/dq071024a-eng.htm> 
TOBIN, Kenneth; GALLAGHER, James J. What happens in high school science classrooms? Journal of Curriculum Studies, 19(6), 549-560, 1987.

Trudel, L. Infuence d'une discussion préalable sur la participation des élèves dans un laboratoire de physique an secondaire. Mémoire de maitrise en éducation. Montréal : Université du Québec à Montréal,1996.

Trudel, L., Parent, C., et Métioui, A. Démarche, cheminement et stratégies : une approche en trois phases pour favoriser la compréhension des concepts scientifiques. Revue des sciences de l'éducation, [s.l], 35(3), 149-172, 2009.

Tuan, H.-L., Chin, C.-C., Shieh, S.-H. (2005). The development of a questionnaire to measure students' motivation towards science learning. International Journal of Science Education, [s.1], 27(6), 639-654, 2005.

WRIGHT, Benjamin D; MASTERS, Geoffrey N. Rating scale analysis. Chicago : Mesa Press, 1982. p. 206. WRIGHT, Benjamin D; STONE, Mark H. Best test design. Chicago : Mesa Press, 1979, p. 222. 
